Archives

$44 \mid 2009$

Varia

\title{
La Chine et son histoire
}

« De la dissolution du veau d'argile dans l'eau de mer »

\section{David Bartel}

\section{(2) OpenEdition}

1 Journals

Édition électronique

URL : http://journals.openedition.org/ccrh/3532

DOI : $10.4000 /$ ccrh.3532

ISSN : $1760-7906$

Éditeur

Centre de recherches historiques - EHESS

Édition imprimée

Date de publication : 25 janvier 2009

Pagination : 91-108

ISSN : 0990-9141

\section{Référence électronique}

David Bartel, «La Chine et son histoire », Les Cahiers du Centre de Recherches Historiques [En ligne], 44 | 2009, mis en ligne le 16 janvier 2012, consulté le 10 décembre 2020. URL : http:// journals.openedition.org/ccrh/3532; DOI : https://doi.org/10.4000/ccrh.3532

Ce document a été généré automatiquement le 10 décembre 2020.

Article L.111-1 du Code de la propriété intellectuelle. 


\title{
La Chine et son histoire
}

\author{
« De la dissolution du veau d'argile dans l'eau de mer»
}

\section{David Bartel}

1 On pourrait répondre au titre du classique de François Dosse, L'histoire en miettes (La Découverte, 1985) par cette belle image de la langue chinoise: «Le veau d'argile entre dans la mer» Niniu ruhai 泥牛入海. Car c'est bien de dissolution qu'il s'agit dans cet article. Comme en Europe et aux États-Unis dix ans plus tôt, la discipline historique en Chine a connu au tournant des années 1990 un phénomène d'implosion, de dissémination. Conséquence directe de la période de réflexion qui suit la répression du mouvement étudiant de 1989, elle est aussi - peut-être surtout - le résultat de la conjonction entre la fin définitive de la foi dans les grandes téléologies positivistes des $\mathrm{XIX}^{\mathrm{e}}$ et $\mathrm{XX}^{\mathrm{e}}$ siècles, et la pénétration en parallèle en Chine de théories historiographiques occidentales, de Michel Foucault (1926-1984) à Edward Saïd (1935-2003).

2 Pour essayer de comprendre l'importance particulière de ce relativisme historique nouveau dans un pays comme la Chine, il faut revenir à la fois aux particularités des liens entre la Chine et son histoire, et aux liens uniques qui unissent les écrivains de l'histoire à l'État chinois. Un double mouvement qui permettra peut-être de mieux interroger les difficultés, les spasmes et les traumatismes que vont laisser la fin des cycles dynastiques au début $\mathrm{du} \mathrm{XX}^{\mathrm{e}}$ siècle et la sortie du marxisme, moins d'un siècle plus tard, avant de laisser place à une instrumentalisation étatique nationaliste et culturelle pas toujours très convaincante. Car en effet, loin d'offrir une richesse de nouvelles perspectives, de nouveaux angles d'attaques, de nouveaux textes et de nouvelles narrations, la « nouvelle » histoire chinoise, n'a que peu l'attrait de la nouveauté. Pendant que quelques thuriféraires de l'historiographie orthodoxe essaient encore à grand peine de vendre la capacité d'adaptation de l'histoire marxiste aux normes nouvelles dans des textes à l'ésotérisme d'un autre temps ${ }^{1}$, les voix de l'histoire officielle semblent bien s'être réapproprié ces nouveaux imports théoriques, pour mieux les phagocyter dans une plainte victimaire antioccidentale sans originalité et intellectuellement peu stimulante, limitant ainsi leur capacité première de résistance aux discours du pouvoir.

3 Pourtant, la vue des chars sur la place Tiananmen pour le soixantième anniversaire de la fondation de la République populaire semble dire autre chose que la légitime fierté 
retrouvée d'un ancien pays - très - pauvre. En fait, cet anniversaire très bien (sur)joué dit deux choses.D'abord, il initie une cohérence historique sans faille qui va désormais de Sun Yat-sen 孙中山 (1866-1925) à Hu Jintao 胡锦涛 (né en 1942) et couvre ainsi tout le XX siècle. Comme dans les cycles dynastiques, au père fondateur succèdent les générations $\mathrm{Hu}$ Jintao représentant la quatrième - dans une geste rendue immuable par une parade de dictature communiste digne de la Guerre froide. Sans rien enlever à la fierté légitime du peuple chinois - tenu bien à l'écart des manifestations - l'exercice de réécriture de l'histoire est évident. L'impressionnante démonstration de force laisse pourtant l'étrange impression d'un pays qui en fait un peu trop, comme pour cacher des incertitudes à venir. Nous reviendrons en fin d'article, avec Michel Bonnin et Jean-Luc Domenach, sur l'interprétation que l'on peut donner de cet anniversaire.

\section{Le modèle dynastique}

4 La Chine très tôt a pris conscience d'elle-même ${ }^{2}$ et très tôt elle a voulu écrire - relater plutôt - son parcours historique. L'Histoire (grand H) y tient le rôle habituellement dévolu à la mythologie ou à la religion. Elle explique la totalité du monde, définit le destin des hommes, et juge de la valeur de la condition humaine ${ }^{3}$. La tradition historique chinoise est un immense corpus de textes dispersés dans les principales bibliothèques du pays. Cette masse de textes, écrits par des secrétaires astronomes liés à un appareil bureaucratique d'État, a cependant une date de naissance. L'acte fondateur de l'écriture de l'Histoire en Chine est celui de Sima Qian 司马迁 (147? - 86? av. J.C.) qui, en 91 avant notre ère, achève la compilation du Shiji 史记, les Mémoires historiques ${ }^{4}$,commencées par son père. Ces annales racontent l'Histoire de Chine depuis le mythique Empereur jaune 黄 帝 Huangdi (2697-2598 av. J.C.). L'ouvrage de Sima Qian - qui avoue s'être inspiré de la démarche de Confucius - est divisé en cinq livres différents (Mémoires, Grandes Familles, Biographies, Chroniques, Livres et Tableaux) et va immédiatement servir de modèle aux générations d'historiens suivantes. L'Histoire sera officielle et contrôlée par le pouvoir. Elle sera ainsi, pendant près de deux millénaires, soumise à trois règles :

5 - relevé quotidien des activités officielles et personnelles de l'empereur sur deux mains courantes, combinées ensuite dans un livre journal ;

6 - à la mort de l'empereur, compilation des trois registres en une Annale véridique de son règne ;

7 - les documents archivés sont interdits d'accès. Ce n'est que la dynastie nouvelle qui pourra raconter l'Histoire officielle de la précédente dynastie ${ }^{5}$.

8 Cette mainmise officielle distingue l'historiographie chinoise des traditions historiennes occidentales sur trois points. D'abord, le découpage de l'histoire en tranches dynastiques sur un modèle stéréotypé est une spécificité chinoise qui a participé à l'impression de cycle attaché aux successions dynastiques. Ensuite, l'existence d'historiens professionnels officiels, salariés et dépendants, fait disparaitre le trait personnel, l'individu derrière le groupe dont il n'est qu'un échantillon. Enfin, l'art traditionnel de la citation, le lourd héritage du génie de la langue va empêcher l'éclosion d'une historiographie critique comparable à la nôtre ${ }^{6}$. Un système tellement éprouvé que le Parti nationaliste 国民党 Guomindang sera tenté après la révolution de 1911 et la fin de l'empire de rédiger l'histoire de la dynastie Qing. Il doit légitimer son pouvoir encore fragile et ne peut interrompre tous les rituels de la tradition impériale. De la même façon qu'il continue à entretenir une 
Cour de dignitaires mandchous - autour de l'empereur Henry Puyi - dont il ne sait trop que faire, sous les auspices du Contrat de bienveillance à l'égard de la cour des Qing, il fonde en 1914 un département chargé de compiler les événements des trois siècles précédents ${ }^{7}$. Ce projet n'aboutira pas et la 清史稿 Qingshigao publiée en 1928 par le Bureau d'historiographie Qing ne sera jamais considérée comme une histoire officielle orthodoxe ${ }^{8}$. Il n'y aura donc en tout que vingt-quatre histoires dynastiques officielles construites sur le même modèle, avec une attention constante et presque monomaniaque à l'institution impériale qui fera dire aux premiers historiens modernes :

Les histoires occidentales enregistrent toutes les politiques d'Etat et les affaires populaires dans le but que le lecteur puisse se faire une idée [des caractéristiques] de l'époque. Les histoires dynastiques chinoises ne se souviennent que des moyens par lesquels une seule famille contrôle le royaume et protège son territoire, en même temps qu'elles louent les offices de ceux qui la servent. La vie du peuple est laissée entièrement de côté. [Les Annales historiques chinoises] ne sont rien d'autre que les généalogies de dix-sept familles impériales. Peut-on appeler ça de l'histoire ? 9

C'est l'intrusion de la puissance occidentale conquérante à la fin du premier tiers du XIX siècle qui va radicalement remettre en question la conception du temps, la relation au passé et donc à l'histoire. Il faut maintenant et dans l'urgence inventer une nouvelle vision globale et créer de toute pièce une histoire qui intègre la Chine au monde tout en lui offrant la possibilité de devenir moderne ${ }^{10}$.

\section{La fin d'un monde}

10 En effet, confrontée à l'efficacité de la puissance matérielle occidentale, la Chine va devoir penser la modernité, prise en tant que formation culturelle du capitalisme. Ce faisant, les intellectuels chinois et japonais vont réviser complètement leur rapport au passé. Pris jusqu'ici comme une décadence progressive par rapport à un mythique Âge d'or, le présent doit désormais offrir la possibilité de la modernité ${ }^{11}$. Dès lors, le changement doit prévaloir sur la stabilité, dans l'urgence créée par l'introduction du darwinisme social en 1896 par Yan Fu 严复 (1853-1924) dans sa célèbre traduction d'Evolution and Ethics de Thomas Huxley (1825-1895) ${ }^{12}$.

11 La période qui ouvre le $\mathrm{XX}^{\mathrm{e}}$ siècle est sans doute un des moments les plus riches et les plus intenses dans les débats d'historiens. Paradoxalement, c'est aussi le délitement progressif de l'État qui va en faire une des périodes de foisonnement intellectuel les plus libres de l'histoire de la Chine moderne. Après la disparition des trois piliers traditionnels de l'empire, le pays est laissé orphelin de sa culture traditionnelle. Quand les examens impériaux, institutionnalisés depuis le VII e siècle, ne sont pas organisés en 1905, c'est l'instrument de reproduction des élites et de conservation de l'orthodoxie idéologique d'État qui disparaît sans faire de bruit. Quand en 1911, le simple soulèvement d'un régiment de Chine centrale entraîne cette étrange "révolution » de Xinhai Xinhai geming 辛亥革命 qui met fin à deux millénaires de cycles impériaux, c'est tout le rapport au temps qui est désormais bouleversé. Moins d'un an plus tard, le calendrier occidental, linéaire, commence à être utilisé en Chine et remplace le temps cyclique, qui se comptait en années de règne de tel ou tel empereur (année $\mathrm{X}$ du règne de l'empereur $\mathrm{Y}$ ) et revenait en début de cycle à chaque succession. Le camp nationaliste invente lui un calendrier qui commence le 10 octobre 1911 et qui est encore utilisé à Taiwan. Quelques années plus tard, quand le chinois classique wenyan 文言, langue administrative, fleuron linguistique 
réservé à l'élite lettrée disparait, au nom de la modernité, au profit de la langue vernaculaire baihua 白话, c'est la dernière clef de voûte de l'ordre d'un monde qui disparaît. C'est dans le vacuum laissé par la fin de ce monde que vont pouvoir s'engouffrer de nouvelles idéologies.

Après l'échec - en 1898 - de la dernière tentative de réforme d'un système dynastique moribond ${ }^{13}$, trois voies vont s'ouvrir pour atteindre la puissance et le confort de la modernité : le nationalisme anti mandchou, le libéralisme et le socialisme. Le libéralisme - politique et économique - que prône un Hu Shi 胡 适 (1891-1962), sous l'influence du pragmatisme du philosophe américain John Dewey (1859-1952), ne répond pas à l'urgence du moment ${ }^{14}$.

Tous les possibles paraissent à nouveau exclus et l'âge du libéralisme apparaît déjà

comme un entracte éphémère entre des siècles de conformisme confucéen et

l'instauration d'une nouvelle orthodoxie,

dit justement Lucien Bianco ${ }^{15}$. L'heure est à la révolution. La victoire bolchevique de 1917 va imposer aux intellectuels chinois le potentiel subversif du marxisme. Leur travail consistera désormais à «couler » l'Histoire dans le moule de l'historiographie marxiste. Faire entrer l'Histoire chinoise dans les cinq phases de développement historique (société primitive, esclavagiste, féodale, capitaliste, socialiste) sera la tâche essentielle des intellectuels chinois des années 1920, et leur lit de douleur pour le demi-siècle à venir ${ }^{16}$.

Il faut bien en effet assimiler la société féodale à un moment pré-moderne pour créer l'inévitabilité de la modernité. Guo Moruo 郭沫 若 (1892-1978) sera le premier à présenter une histoire complète conforme à ce modèle dans ses Études de la société chinoise ancienne publiées en 1930, puis traduites en partie plus tard ${ }^{17}$. Adopté par le Parti communiste au $6^{\text {ème }}$ congrès de 1928, ce modèle devient l'étalon de l'histoire officielle. Il s'ensuivra de longs débats sur le féodalisme, le mode de production asiatique, les causes premières et les créateurs de l'histoire. Ce qui compte, c'est que l'universalisme marxiste permet désormais de dépasser l'Occident et de rattacher la Chine à un schéma qui transcende la dualité Chine/Occident en rattachant la Chine au monde moderne.

La guerre sino-japonaise va interrompre les débats à partir de 1937, et les énergies des intellectuels vont être détournées vers la propagande et la résistance nationale. Cependant, les dés sont jetés et la fondation de la République populaire en 1949 conservera le paradigme de Guo Moruo. Désormais, les grandes lignes ne bougeront plus et les quelques champs laissés aux recherches historiennes ne seront plus que d'indispensables et salutaires exercices de gymnastique intellectuelle. Aucune conclusion ne devant plus sortir du dogme, aucune grande nouveauté ne pourra émerger ${ }^{18}$. Seules d'importantes découvertes archéologiques feront avancer les recherches historiques en Chine ${ }^{19}$. Il faudra attendre 1976 et la mort de Mao Zedong 毛泽东 (1893-1976) pour que renaissent les études historiques.

\section{L'histoire en République populaire}

16 Ainsi,

L'école d'historiographie dominante, influencée par le marxisme orthodoxe et les schémas staliniens de «développement universel » formulé dans les années 1920, insiste sur le rôle déterminant de forces économiques impersonnelles pour expliquer la persistance d'une autorité despotique dans l'histoire impériale et moderne de Chine. Pour ces historiens que l'on dit «matérialistes », la Chine a fait 
l'expérience relativement continue et linéaire de formation du despotisme, de Qin

Shihuang 秦始皇 (259-210 avant J.C.) à Mao Zedong ${ }^{20}$.

cadre dans lequel la jeunesse chinoise née avec la République popu-laire va grandir. Le dogme, qui peu à peu se transforme en foi indiscutable, se forme autour de quelques croyances que les historiens communistes vont tourner et retourner sans possibilité de salut. Quelles que soient leurs conclusions, elles ne devront pas dévier de la doctrine. Outre les cinq étapes du développement historique de l'Histoire vues ci-dessus, les historiens vont se voir contraints d'octroyer aux masses paysannes un rôle central ${ }^{21}$. En plus d'être les forces motrices de l'Histoire, les «faiseurs de l'histoire» (Lishi de chuangzaozhe Lishi de chuangzaozhe 历史的创造者), elles deviennent la justification unique de la théorie marxiste de la lutte des classes ${ }^{22}$. Dans la volonté non dissimulée de s'intégrer à l'Histoire-Monde, et de bien marquer l'irréconciliable déconnexion avec la période impériale, il faut désormais mettre en avant les vocables de luttes, de conflits, de guerres paysannes, de révoltes et de révolutions. Une emphase qui volon-tairement tourne le dos au but traditionnel de la politique chinoise: l'harmonie ${ }^{23}$. (Le projet politique de "Petite prospérité » (Xiaokang shehui 小康社会) et l'abandon officiel du projet révolutionnaire peuvent être, dans cette perspective, interprétés comme la reprise d'un projet politique national déjà ancien, après quelques décennies de déviation). Cette volonté de faire de la révolte paysanne la base de l'ordre vient continuellement butter sur le proverbe populaire : « Les perdants restent des brigands, les vainqueurs deviennent des rois» (Baizhe wei kou, chengzhi wei wang 败者为寇，成者为王). Un proverbe qui se lit de deux manières également contradictoires avec l'orthodoxie nouvelle : pour réussir et établir effectivement un gouvernement, le ou les paysans victorieux devront s'allier avec l'aristocratie en place et devenir un instrument de la classe dirigeante; ou, une fois en place, le nouveau gouvernement se transformera progressivement en une nouvelle aristocratie. Dans les deux cas, on le voit - les seuls répandus dans l'histoire chinoise des mouvements paysans - l'institutionnalisation de la révolte, si elle réussit, reconduira la société féodale dans sa structure initiale. En cas d'échec, le rebelle reste un bandit ${ }^{24}$. Cette contradiction entre volonté idéologique et réalité historique ne sera jamais dépassée, mais les paysans sont désormais les maîtres du pays « officiel». Avec l'ère Mao sonne l'heure de gloire du mythe paysan dans une reconstruction a posteriori de l'Histoire.

\section{Dégel et impasse}

La Chine en 1976 n'est plus que l'ombre d'elle-même. Silence, peur, lassitude et pénurie sont le lot quotidien de la majorité ${ }^{25}$. Deng Xiaoping 邓小平 (1904-1997) lance alors sa bataille pour reprendre le pouvoir et sauver ce qui peut encore l'être. Pour cela, il a besoin de construire une alliance entre réformateurs du sérail et des classes populaire exténuées par trois décennies de volontarisme politique et de revirement idéologique. Il laisse volontairement le contrôle se fissurer, et s'amoindrir les risques encourus par l'hétérodoxie idéologique. Deng le sait, il a besoin du soutien des intellectuels pour penser sa réforme ${ }^{26}$.

Cependant, au monde des historiens correspond un complexe de problèmes particuliers. Les écrivains de l'histoire se retrouvent très vite neutralisés par de multiples entraves. D'abord, ceux qui sont toujours là après trente ans de maoïsme sont bien conscients du privilège de leur situation. Ils en connaissent aussi la fragilité et hésitent à sortir du cadre

Les Cahiers du Centre de Recherches Historiques, 44 | 2009 
dans lequel ils ont appris à survivre. Ensuite, l'habitude qu'ils ont prise de se perdre infiniment dans une vulgate marxiste ésotérique sans aboutir à de réelles conclusions empêche toute avancée théorique d'importance. Enfin, et peut-être surtout, quarante années de gangue intellectuelle ont si bien formaté les esprits, qu'il leur est impossible de sortir du cadre conceptuel de leur formation. Ils sont victimes des pesanteurs de l'éducation qu'ils ont reçue ${ }^{27}$. En terme de génération aussi se creuse un important fossé entre les plus anciens - ceux qui restent - et qui ont massivement cru dans l'utopie des premières années du régime contre le despotisme et la Chine "féodale». Il leur est difficile de renier leur engagement de jeunesse au coté de la «Chine nouvelle». Ils savent aussi parfaitement ce qu'ils risquent. Les historiens les plus jeunes, eux, n'ont connu que cette «Chine Nouvelle», sa fermeture, son rigorisme et ses contraintes. Élevés dans un monde clos où tout est politique, contraignant, uniforme, il est difficile pour les jeunes générations d'inventer une nouvelle conception de l'histoire. À la fin des années 1970, la profession d'historien n'arrive pas à se défaire d'une "ossification de la pensée " particulièrement résistante et à définir de nouveaux horizons ${ }^{28}$.

Ce n'est donc pas un hasard si le vent libérateur viendra de l'extérieur de la profession. Jin Guantao est scientifique de formation. Liu Qingfeng, sa femme est écrivain et historienne. Ils publient en 1979, d'abord sous le manteau, un essai où ils tentent de systématiser un ensemble de réflexions nées dès le début de la décennie, dans le feu de la Révolution culturelle. L'essai s'intitule Prospérité et crise: à propos de l'hyperstabilité des structures de la société féodale chinoise et met à mal toute la structure orthodoxe de l'histoire chinoise ${ }^{29}$. Sa lecture et la polémique qu'il déclenche relancent le débat sur l'histoire et participent d'une indispensable décongestion intellectuelle.

\section{En marche vers le futur}

En 1979, quand est publié Prospérité et crise pour la première fois, son audience est encore confidentielle et la Chine encore trop engourdie pour réagir. Les auteurs sont critiqués deux fois malgré tout, d'abord pendant la campagne " contre le libéralisme bourgeois " en 1981, et ensuite pendant celle « contre la pollution spirituelle » en 1983. La polémique ne s'inscrit pas encore cependant dans l'enjeu politique et idéologique du moment ${ }^{30}$. Cinq années après sa première publication, pour pouvoir diffuser l'essai sur une autre échelle, Jin et Liu fondent la collection 《En marche vers le Futur» Zouxiangweilai走向未来et publie une version simplifiée de leur hypothèse historique ${ }^{31}$. Sa réception bénéficie d'une couverture nouvelle. En 1984, les débats entre réformateurs et vieille garde conservatrice ont pris un nouveau visage. Les clivages au sein de la réforme denguiste sont désormais clairement marqués entre les conservateurs réunis autour de l'économiste "immortel » Chen Yun 陈云 (1905-1995) et les réformateurs en rang derrière Hu Yaobang 胡耀邦 (1915-1989) puis Zhao Ziyang 赵紫阳 (1919-2005). Le timing cette fois est parfait. Les critiques fusent de toutes parts et soulèvent la curiosité d'une Chine qui a soif de connaissances et de nouveauté. Cette rencontre entre un public et une collection va assurer un succès commercial à une construction financière à la limite de la légalité socialiste ${ }^{32}$. Un succès qui va se répandre à tout le pays et servira de modèle à la « Fièvre des collections »Congshure 丛书热 qui s'annonce ${ }^{33}$. Dans la première série de publication, outre Prospérité et crise, on trouve des traductions de Douglas Hofstadter, de Fritjof Capra et de Max Weber, ainsi qu'une traduction du fameux rapport du Club de Rome : Les limites de la croissance. Enfin, de jeunes intellectuels chinois trentenaires y font aussi leurs 
premières armes ${ }^{34}$. Dans les librairies, on frôle l'émeute. Les séries de livres sont réimprimées. Le succès est là. Le marché existe.

\section{L'histoire en débat}

Même si l'hypothèse de l'hyperstabilité semble accepter l'idée d'une évolution de l'histoire procédant par stades, elle oublie l'aspect téléologique fondamental de l'orthodoxie marxiste. L'histoire doit être en marche vers quelque chose, le paradis communiste. Or, le schéma que proposent Jin et Liu n'impose pas de finalité. De l'abandon du déterminisme économique découle en effet un enchaînement de circonstances où s'effondrent les uns après les autres une série d'habitus théoriques supposés inébranlables ${ }^{35}$. Quand Jin et Liu parlent de développements internes de trois sous-ensembles - idéologique, politique et économique - aucun n'a de prépondérance sur l'autre. Le déterminisme de Jin Guantao n'est plus économique, et la recherche des causes premières n'a plus que peu d'intérêt. Les fins sont indéterminables et dépendent constamment des interactions - stimuli et rétroactions, ou feedback - entre sous-ensembles. Les historiens orthodoxes lui reprochent de brandir un nouveau drapeau pour remplacer celui du marxisme et d'opposer la science exacte à la méthodologie fondamentale marxiste ${ }^{36}$. En sortant des sempiternels débats sur la périodisation et la causalité, Jin Guantao et Liu Qingfeng confirment pour la première fois aussi publiquement et méthodiquement le déclin du modèle orthodoxe. L'hypothèse s'attaque aussi au fondement scientifique du discours idéologique. En prouvant "scientifiquement" l'existence de cycles périodiques de destruction des progrès de la civilisation chinoise, Jin et Liu sapent deux principes de l'historiographie officielle, le progrès linéaire de l'histoire et la force motrice de la classe paysanne dans l'histoire de la Chine féodale ${ }^{37}$. Ce dernier argument, véritable crime de lèse-majesté, met l'accent sur le caractère endogène du sous-développement chinois et dédouane les interventions étrangères (mongole, mandchoue et occidentale) pour la plus grande fureur des thuriféraires du Parti ${ }^{38}$. Plus généralement, les penseurs humanistes voient dans l'effort de modélisation de Jin Guantao une évacuation radicale du facteur humain. On trouve ici la même critique que celle faite régulièrement à Fernand Braudel et à ses tentatives d'histoire totale : la mise à l'écart du facteur humain, écrasé par le poids de structures dont il ne peut, au mieux, que prendre conscience ${ }^{39}$. Les sociologues, davantage portés sur l'art du modèle, y trouveront un exercice intellectuellement stimulant ${ }^{40}$. Les critiques viennent de Taiwan et de Hongkong, et s'exacerbent en même temps que les tensions entre pouvoir et société se tendent ${ }^{41}$. Les critiques arrivent aussi d'Occident, où la vogue structuraliste a passé ${ }^{42}$. Tour à tour, Jin et Liu sont accusés d'être les fondateurs d'une "cybernétique historique» ou d'être des «historiens structuralistes ». Ils refusent toutes ces simplifications. Si effectivement, ils revendiquent l'inspiration intellectuelle de la cybernétique, ils en dépassent le strict cadre dès qu'interviennent les concepts d'asystémies ou d'aliénation. De même, s'ils reconnaissent le lien avec le structuralisme dans l'ambition d'aborder la globalité de la civilisation chinoise, de regarder une organisation comme un tout nécessitant pour survivre des interactions entre ses parties, ils refusent l'étude synchronique du structuralisme. Ils sont eux intéressés par les évolutions de l'ensemble ${ }^{43}$. 


\section{Jaune et bleu : L'élégie du fleuve}

23

Jin Guantao travaille avec quelques intellectuels de sa génération à un nouveau projet. Il s'agit désormais de toucher un public infiniment plus large que celui des collections de livres - majoritairement étudiants et intellectuels - grâce à un nouveau médium, la télévision. Il s'agit d'introduire une audience de masse à quelques uns des débats hétérodoxes qui agitent un monde académique peu enclin d'habitude à partager ses découvertes avec le grand public dans un mariage inhabituel de média de masse et d'académisme pop ${ }^{44}$. Jin Guantao s'applique avec des activistes de sa génération ( $\mathrm{Su}$ Xiaokang 苏晓康 (né en 1949), Xia Jun 夏骏 (né en 1962), Wang Luxiang 王鲁湘 (né en 1956), Wang Juntao 王军涛 (né en 1958), Bao Zunxin 包尊信 (1937-2007)) à l'écriture d'une série télévisée. Ils veulent, via un médium extrêmement populaire, exposer l'état de leurs réflexions sur l'histoire et la civilisation chinoise. Grâce aux complicités de quelques cadres intermédiaires plus âgés au sein de CCTV (Chinese Central Television), le groupe réalise les six épisodes de Heshang 河歾 (L'élégie du fleuve). La série est diffusée nationalement du 11 au 16 juin 1988. En même temps, le Quotidien du peuple en publie le script ${ }^{45}$. Le succès émotionnel de l'émission dépasse toutes les attentes ${ }^{46}$. Les limites étroites du débat académiques sont brisées. La production est envahie de sacs de courrier - éloges, critiques ou consternations ${ }^{47}$ - venant des quatre coins du pays ${ }^{48}$. Les titres des six épisodes sont volontairement dramatiques : À la recherche d'un rêve, Destinée, Lumières spirituelles, Nouvel Âge, Crise, Le grand bleu. Les auteurs y opposent à une "Chine Jaune", Chine du nord, berceau traditionnel d'une civilisation sclérosée, introvertie et immobile, une "Chine bleue", inventive, ouverte sur le monde, la science et la démocratie. Nos jeunes intellectuels présentent les habituels symboles de puissance que sont le Dragon, le Fleuve jaune ou la Grande muraille comme les origines d'une double soumission qui a enlisé le pays dans une utopie paysanne et patriarcale. Sous la critique de la culture traditionnelle, l'attaque contre la Chine socialiste est évidente. La domination de la nature indomptable du fleuve jaune se reflète politiquement dans une immémoriale domination despotique ${ }^{49}$. Si l'unité de l'œuvre est d'ordre symbolique, elle n'est pas conceptuelle. Les raccourcis pris avec la réalité historique seront beaucoup reprochés aux auteurs ${ }^{50}$. Mais, l'exhaustivité académique n'est cependant pas le but recherché. L'enjeu est ailleurs. Il s'agit d'inventer le grand récit qui saura affranchir les esprits du grand récit marxiste. Et, malgré leurs erreurs, les collaborateurs de Jin Guantao et Liu Qingfeng ont fait entendre leurs voix comme jamais un groupe dissident ne l'avait fait auparavant en République populaire. Un fossé se creuse entre une base qui veut accélérer le pas des réformes et un sommet où la faction réformatrice est affaiblie depuis le $13^{\text {ème }}$ congrès $d u$ Parti et la destitution de Hu Yaobang ${ }^{51}$. La série est devenue l'enjeu d'une lutte au sommet entre partisans et adversaires de l'ouverture. L'année 1989 restera pour Su Xiaokang l'Année du cri ${ }^{52}$. C'est en effet l'année de toutes les commémorations - fondation de la République, mouvement du 4 mai, bicentenaire de la Révolution française - et par conséquent elle sera l'année de tous les dangers ${ }^{53}$.

\section{Le paradoxe des Lumières chinoises}

Il est intéressant de noter que la série Heshang dépasse entièrement son propos. En offrant à une masse immense de spectateurs une certaine vision de l'histoire chinoise, les 
auteurs lui font partager la conscience qu'ils ont des urgences qui pressent le pays. Ils en décrivent les causes, et en " hurlent » les solutions. Ainsi, il transparaît tout au long des six épisodes que les auteurs de la série sont plutôt bienveillants avec le projet néoautoritaire prôné par la faction réformatrice de Zhao Ziyang ${ }^{54}$. Ce faisant, on peut mettre à jour dans ce soutien politique les paradoxes dont souffrent les intellectuels en Chine. En effet, dans la lecture que donnent les historiens engagés dans la série, la construction qu'ils donnent du passé impérial souffre, même inconsciemment, de la nostalgie d'un âge d'or. Ce faisant, le discours que proposent ces jeunes intellectuels trahit la cause des Lumières de libération de l'individu et sombre dans un nationalisme dont on sent bien la difficulté qu'ils ont à se distancer et qui transpire dans toute les années quatre-vingt. Élitistes et modernes, ils font le même rêve que leurs précurseurs confucéens, celui d'un État prospère, puissant, hégémonique. Ils envisagent cet État exactement comme ils envisagent l'empire qu'ils dénoncent: une structure monolithique abstraite extérieure aux hommes qui forment la nation. Un horizon bleu qui ravivera dans le futur un passé glorieux. L'usage fait du symbole du dragon dans la série est à ce titre révélateur. Dès le premier épisode, ce symbole couramment attaché au monde chinois, emblème du pouvoir et de la superbe impériale est transformé en animal abominable, insigne de l'autocratie traditionnelle. Ce même dragon revient à nouveau dans le sixième épisode de la série, cette fois sous la forme d'un puissant symbole de modernité, un "gigantesque dragon économique ». Ainsi, il ne s'agit pas comme beaucoup l'ont affirmé dans les critiques virulentes contre la série, de nihilisme national, mais d'un sentiment immensément plus complexe ${ }^{55}$. Les années quatre-vingt sont marquées par l'ambivalence d'une avant-garde intellectuelle qui honnit autant qu'elle révère son propre héritage culturel et national ${ }^{56}$.

\section{French theory et orientalisme}

Avec la répression du mouvement étudiant le 4 juin 1989 s'amorce une période de réflexion plus académique sur les problèmes posés par l'histoire de Chine. En même temps, le nationalisme va servir de recours à un pouvoir idéologiquement exsangue. Quand Deng Xiaoping relance la réforme économique en 1992, la Chine va rapidement rattraper le rythme du monde dans de nombreux domaines. En histoire, si des écrivains comme Jean-François Lyotard (1924-1998) ou Michel Foucault avaient bien été traduits dès le début des années quatre-vingt grâce notamment aux efforts continus de Yue Daiyun 乐黛云(1931-2008), c'est au début de la décennie suivante que l'incrédulité qu'ils proposent à l'égard des grands récits va prendre en Chine tout son sens ${ }^{57}$. En même temps que l'Histoire (H majuscule, singulier) s'émiette, pour devenir des histoires, les conférences données par l'historien marxiste Fredric Jameson en Chine au milieu des années quatre-vingt ont un succès considérable ${ }^{58}$. Il faudra attendre les années quatrevingt-dix pour que la "crise de l'historicité ", conséquence de l'indigénisation 本土化 Bentuhua des discours historiens liés aux lectures postcoloniales rendent l'objet historique désormais extrêmement difficile à définir. À ce propos, l'influence de la lecture en Chine des écrits d'Edward Saïd ne peut être sous-estimée. Car cette indigénisation crée une "sinité » dont le discours se veut représentatif du Tiers-Monde contre l'hégémonie occidentale. Au contraire du projet foucaldien qui cherchait à lire et entendre les langages du pouvoir présent partout - école, hôpital, prison, usine - les discours postmoderne et postcolonial se retrouvent ici instrumentalisés par un ethnocentrisme qui vient renouveler un discours nationaliste aux relents populistes parfois xénophobes, 
autant qu'il garde les critiques internationales à la distance qu'impose désormais le relativisme historique et culturel ${ }^{59}$. C'est d'ailleurs la raison pour laquelle les théories critiques postcoloniales et leur mariage avec la «logique de la blessure » rencontre indéniablement en Chine plus de succès que les théories de la postmodernité ${ }^{60}$. Ainsi, on peut croire que la percée en Chine de ces théories critiques et sa récupération nativiste correspond aussi à la période de vide idéologique qui suit la mort des idéaux socialistes sur la place Tiananmen en 1989. Les autorités trouvent dans la réactualisation du discours nationaliste l'instrument fédérateur que l'on sait ${ }^{61}$.

Cependant, loin de n'être qu'une logorrhée du discours, critique, littéraire ou historien, il existe aussi dans l'immense production des «postologues » des techniques de résistances où, masqué, le « post » qu'il soit moderne ou colonial ne veut dire que post-1989. La date du 4 juin étant effacée de la mémoire collective, ceux qui s'en souviennent ont dû inventer des stratégies pour continuer à produire du savoir. Il y a dans ce glissement de signifiant, le reflet et la contribution aux relations de plus en plus complexes et instables entre nouveaux discours et idéologie officielle, car talking post is talking past. Et tous les discours « post » naissent le même jour, le 4 juin 1989. Pour Xu Ben, la question n'est pas de savoir si ces théories doivent être utilisées ou pas, mais bien comment faire correspondre leur acuité et leur pertinence à un agenda progressiste et émancipateur, en un mot démocratique ${ }^{62}$. Car dans l'idée d'une continuité chronologique, le post suppose un après, mais après quoi ? Après le colonialisme ? La Chine n'a pas été colonisée. Elle a su conserver sa langue, invalidant ainsi un des points fondamentaux des lectures postcoloniales, celui d'être composées de textes pensés et écrits dans l'idiome de l'envahisseur ${ }^{63}$. Et même si l'impact des concessions - Shanghai surtout - a été décisif dans l'idée que les intellectuels se sont fait de la modernité, il ne s'agit que d'une construction mentale née dans les cabarets ou les cafés littéraires, sous les platanes de l'avenue Foch de la concession française et dans les distractions qu'offraient le grand magasin Wing On sur la rue de Nankin ${ }^{64}$. La question du lien entre la Chine du XX $\mathrm{X}^{\mathrm{e}}$ siècle et le concept de modernité est un point central de la réflexion des historiens chinois aujourd'hui ${ }^{65}$. Et, entre occidentalisation, nativisme et relativisme, la question de la modernité chinoise n'est pas près d'être évacuée. Car, malgré l'attrait des postologies en Chine, la question de l'adéquation de la Chine au postmodernisme comme « logique du capitalisme tardif » n'a toujours pas trouvé de réponse.

\section{Conclusion : passé flou, futur incertain, présent ambigu}

Alors que le retour victorieux sur la scène internationale semble enfin réconcilier ce vieux pays avec l'histoire douloureuse des siècles récents, l'amnésie nationaliste orchestrée par la main mise de l'État-parti sur l'histoire du pays et sur tous les médias semble bien prouver qu'au contraire, l'actuelle génération de dirigeants semble se crisper dans un rigorisme orthodoxe d'un autre temps. Les parades récentes pour le $60^{\text {ème }}$ anniversaire de la fondation de la République Populaire de Chine semblent bien confirmer que pendant que le Parti-État s'auto congratule et bombe le torse devant les caméras du monde entier pour montrer sa puissance enfin retrouvée, le peuple chinois est tout bonnement exclu des célébrations. Si le Parti communiste chinois (PCC) est fier de sa réussite, de son maintien au pouvoir, il donne le sentiment d'avoir peur de ce peuple qu'il est pourtant censé représenter ${ }^{66}$. Ce défilé n'est-il pas finalement celui de la morgue 
d'une classe au pouvoir, affranchie de tout contrôle, de toute limitation dans ses ambitions? N'assistons-nous pas au couronnement d'une forme nouvelle de bonapartisme, mélange d'élitisme et de nationalisme, saupoudré de consultation populaire?

C'est Tu Wei-Ming 杜维明 (né en 1940) célèbre professeur d'histoire et de philosophie à Harvard, dans l'introduction d'un ouvrage récent qui nous rappelle à la fois le rôle des historiens et les spécificités chinoises de cette profession. Il insiste sur l'amnésie qui marque l'histoire du $\mathrm{XX}^{\mathrm{e}}$ siècle en Chine et sur la volonté du gouvernement de ne regarder que vers l'avenir, pour mieux transformer le passé en récit hagiographique d'où n'émergent que la résistance antijaponaise, et la montée du Parti communiste chinois instrumentalisées à outrance pour mieux masquer la vacuité idéologique du Parti et la fragilité du système politique en s'aventurant sur un terrain nationaliste fertile, mais risqué. Le refus obstiné d'autoriser les recherches sur la Révolution culturelle et la répression violente du 4 juin 1989 sont pour lui une indication claire que la tentation politique d'effacer les mémoires collectives est une pièce majeure du contrôle idéologique en Chine contemporaine ${ }^{67}$.

\section{NOTES}

1. Il faut lire un Jiang Dachun 蒋大椿 (né en 1940) du département d'histoire de l'Académie des sciences sociales de Chine (ASSC), qui n'a jamais dévié de la ligne officielle. Voir par exemple, en anglais, «Contemporary Chinese Approaches to Historical Research and the Development of the Marxist Conception of History ", Chinese Studies in History, Vol.38, printemps/été 2005, p. 114-157.

2. Edward Wang, Historical Writings in 20th Century China: Methodological Innovation and Ideological Influence, Rowan University. Disponible sur le Web [http://www.oslo2000.uio.no/program/ papers/s1/s1-wang.pdf] (page consultée le 18 juin 2007).

3. Pierre Ryckmans, in Encyclopaedia Universalis, Dictionnaire de la civilisation chinoise, Paris, Albin Michel, 1998, p. 649-650.

4. Edouard Chavannes a traduit 47 chapitres des Annales Historiques publiés en 6 volumes entre 1895 et 1905. Disponible sur le Web : [http://classiques.uqac.ca/classiques/sema_tsien/ sema_tsien.html] (Page consultée le 18 juin 2007).

5. Léon Vandermeersch, «La conception chinoise de l'histoire » in La pensée en Chine aujourd'hui, Anne Cheng (dir.), Paris, Gallimard, 2007, p. 50-52.

6. Étienne Balazs, La bureaucratie céleste, Paris, Gallimard, 1968, p. 47.

7. Henry Puyi, J'étais empereur de Chine, Paris, Flammarion, 1975, p. 32-33. Sur les relations entre la cour des Qing et la toute jeune république chinoise, voir David Bartel «Pourquoi la Cité interdite est-elle vide?", in Chine: Art, propagande et résistances, Emmanuel Lincot (éd.), Paris, You-Feng, 2007.

8. Hsi-yuan Chen, «Last chapter unfinished: The making of the official Qing History and the crisis of Traditional Chinese Historiography ", in Historiography East and West, Leyde, Brill Academic Publisher, vol.2, 2006, p. 173-204.

9. Renzhu Xu en 1889, cité par Timothy Brook, «Capitalism and the Writing of Modern History in China ", in China and Historical Capitalism: Genealogies of Sinological Knowledge, Timothy Brook \& 
Gregory Blue (dirs.), Cambridge (Mass.), University Press, 1999, p.127. Il faut quand même noter ici une forme d'illusion des jeunes historiens chinois sur la qualité des historiens occidentaux. En France, par exemple, l'histoire à la même époque est encore majoritairement une histoirebataille, positiviste, fortement nationaliste, totalement revancharde vis-à-vis de l'Allemagne après la défaite de 1871. Voir François Dosse, L'histoire en miettes, Paris, La Découverte, 1987.

10. Timothy Brook et Gregory Blue, op. cit., p.125.

11. Arguments développés par Timothy Brook, «Capitalism and the Writing of Modern History in China », in Timothy Brook et Gregory Blue (dir.), Ibid. p. 110-157.

12. Thomas Huxley, Evolution and Ethics, Oxford, The Romanes Lectures, chapitre 9 de la Collection des essais, 1893 ; la célèbre traduction de Yan Fu de 1896 est disponible sur le Web : [http://rwxy.tsinghua.edu.cn/rwfg/ydsm/ydsm-qw/00104/004.htm] (Visité le 12 juin 2007).

13. Même si des recherches récentes relativisent les conséquences de la Réforme des cent jours et révisent ses résultats de manière positive, c'est encore un constat d'échec qui entoure globalement cette tentative de réforme avortée par un coup d'état conservateur. Voir Rethinking the 1898 Reform Period: Political and Cultural Change in Late Qing China, Rebecca Karl et Peter Zarow (eds.), Cambridge, (Mass.) et Londres, Harvard University Press, 2004.

14. John Dewey, Comment nous pensons, Paris, Empêcheurs de penser en rond, 2004.

15. Lucien Bianco, Les origines de la révolution chinoise, Paris, Gallimard, 2007, p. 95.

16. La genèse détaillée de la structuration des cinq phases, Brook Timothy, op. cit., p. 133.

17. Guo Moruo, Études sur la société ancienne, Pékin,Renmin chubanshe, 1977.

18. Maurice Meisner, "Treatment of Materialist Conception of History ", in History in Communist China, Albert Feuerwerker (éd.), Cambridge (Mass.), MIT Press, 1968, p. 304-305.

19. Te-K'un Cheng, "Archeology in Communist China ", in ibid., p. 55 ; etJocelyne Fresnais, La protection du patrimoine en République Populaire de Chine (1949-1999), Paris, Éditions du CTHS, 2001, p. 27-40.

20. Laurence Sullivan, "The Controversy over Feudal Despotism ", in Using the Past to Serve the Present, Jonathan Unger (éd.), Michigan Uniniversity Press, M.E.Sharpe, 1993, p. 175 (traduction de l'auteur).

21. Rôle central qui sera contesté dès 1967 par la sinologie française. Voir Lucien Bianco, Les origines de la révolution chinoise, Paris, Gallimard, 2007 (première édition 1967).

22. James Harrison, "Interpretations of Chinese Peasant Wars ", in Feuerwerker (ed.), op. cit., p. 190.

23. Ivan P. Kamenarović, Le conflit. Perception chinoise et occidentale, Paris, La Nuit Surveillée, Le Cerf, 2001, p. 57-58.

24. James Harrison, op. cit., p. 194-196.

25. Cheng Yingxiang, Dégel de l'intelligence en Chine, Paris, Collection « Témoins », Gallimard, 2004, p. 26-27.

26. Chen Yan, L'éveil de la Chine, La Tour d'Aigues, éditions de l'Aube, 2002, p. 32.

27. Hans-Günther Herrmann, «Aspects théoriques de l'historiographie chinoise des années 1980 »,Études chinoises, Vol. X, n¹-2, Paris, AFEC, printemps-automne 1991, p. 167.

28. Françoise Lauwaert, Un hiver à Pékin, Revue belge d'histoire contemporaine,Bruxelles, vol. 9, n ${ }^{\circ} 3-4,1978$, p. 578-581. [http://www.flwi.ugent.be/btng-rbhc/pdf/BTNG-RBHC,\%2009,\%201978, \%203-4,\%20pp\%20565-582.pdf] (visité le 22 septembre 2009).

29. Jin Guantao et Liu Qingfeng, Xingsheng yu Weiji - lun Zhongguo fengjian shehui de chaowending jiegou 兴盛与危机一论中国封建社会的超稳定结构 [Prospérité et crise : l'Hyperstabilité des structures de la société féodale chinoise]. Publié sous forme de manuscrit dés la fin des années soixante-dix, le texte sera distribué par les Éditions Populaires du Hunan, puis du Sichuan à partir de 1984. L'essai sera publié ensuite à différentes reprises à Taiwan en 1989, 1992 (Fengyun Shidai), et à Hongkong (Hongkong Chinese University Press) en 1998. Une dernière édition remaniée est sortie chez Fengyun Shidai en 2005. 
30. Yan Chen, L'Éveil de la Chine, op. cit., p. 88.

31. Jin Guantao et Liu Qingfeng, Zai lishi de biaoxiang beihou在历史的表象背后 [Derrière l'apparence de l'Histoire] dans la série Zouxiangweilai, Sichuan renmin chubanshe, 1984.

32. Sur la structure administrative de la collection, Chen Fong-ching \& Jin Guantao, From Youthful Manuscripts to River Elegy, Hong Kong, Chinese University Press, 1997, p. 95-108.

33. Chen Yan, op. cit., p. 89-91.

34. Douglas Hofstadter, Gödel, Escher, Bach, Paris, Interéditions, 1985 ; Fritjof Capra, Le tao de la physique, Paris, Tchou, 1979; Max Weber, L'Éthique protestante et l'esprit du capitalisme (traduction de J. Chavy), Paris, Plon, 1964 ; Donnela et Dennis Meadows, Jorgen Randers et William Behrens, Halte à la croissance ? Rapport sur les limites de la croissance, Paris, Fayard, 1972 ; Wang Xiaoqiang et Bai Nanfeng, The Poverty of Plenty, Basingstoke, Macmillan, 1991.

35. David Kelly, "Out of the Abyss : the Ironic Marxism of Jin Guantao », Actes de séminaires non publiés, Canberra,Australian National University, novembre 1988.

36. Sur cette polémique: Bai Gang, Zhongguo fengjian shehui changqi yanxu wenti lunzhan de youlai $y u$ fazhan中国封建社会长期延续问题论战的由来与发展 [Origine et développement du débat sur la question de la longue durée de la société féodale en Chine], Pékin, éditions des sciences sociales chinoises, 1984. Cité par Chen Yan, op. cit., note 88, p. 87.

37. Chen Yan, op.cit., p. 88.

38. Hans-Günther Herrmann, art. cité.

39. François Dosse, L’histoire en miettes, Paris, La Découverte, 2005 (1 $1^{\text {ère }}$ édition 1985), p. 155.

40. Daniel Kane, "Jin Guantao, Liu Qingfeng and their Historical Systems Evolution Theory ", Papers on Far Eastern History, $n^{\circ}$ 39, Australian National Universtity, mars 1989, p. 63-64.

41. Lu Fanzhi, Zhongguo wenhua fazhan xingtai yu « Yaxiya shengchan fangshi 中国文化发展形态与 《亚细 亚生产方式» [Le développement de la culture chinoise et le «mode de production asiatique»], Appendice 1: Guanyu Zhongguo wenhua jiegou yanjiu de taolun - ping Jin Guantao, Liu Qingfeng de Xingsheng yu Weiji yu Zai lishi biaoxiang beihou关于中国文化结构研究的讨论一评金观涛，刘青峰的“兴盛与危机” 与“在历史表象背后” [Discussion des recherches sur la structure de la culture chinoise : une critique de Prospérité et crise et de Derrière l'apparence de l'Histoire de Jin Guantao et Liu Qingfeng], Hongkong, Jingying chuban gongsi, 09.1983 ; Guan Donggui, Huiping Xinsheng yu weiji 回评兴盛与危机 [Lecture critique de Prospérité et Crise], Taibei pinglun 4, 1988 ; Hu Changzhi, Guoqu de miwang ping Xingsheng yu weiji 过去的迷惘一评“兴盛与危机” [Incertitude du passé : critique de Prospérité et crise ]Wenxing, Taiwan, 03.1988 ;He Zhaowu, Lishi yanjiu de yige jia wenti - cong suowei Zhongguo fengjian shehui de changqi tingzhilun shuoqi历史研究的一个假问题一从所谓中国封建社会的长期停滞论说起[Un faux problème de la recherche historique : sur la dite théorie de la stagnation prolongée de la société féodale chinoise], Baike zhishi, 1989 ( ${ }^{\circ} 5$ p. 6-8) ou Jiang Dachun, Jin Guantao de lilun zhuiqiu jiqi $m i w u$ 金观涛的理论追求及其迷误 [Le zèle théorique de Jin Guantao et ses erreurs], Pékin, Jindaishi yanjiu, 1990 (n² 2, p. 10-30).

42. Il faut quand même noter la coïncidence du premier livre de Jin Guantao avec cette systémique de la crise qui a jeté, aussi en Occident, et à la même époque les bases d'une contreutopie décisive. En Occident, c'est le début du tout communicationnel, en Chine, c'est la confirmation d'une foi renouvelée dans le rationalisme scientifique. Voir François Cusset, La décennie, Paris, La Découverte, 2006.

43. Daniel Kane, op. cit., p. 64.

44. Geremie Barmé, "History for the Masses ", in Using the Past to Serve the Present, Jonathan Unger (ed.), op. cit., p.279.

45. Jin Guantao \& Chen Fong-ching, >From Youthful Manuscripts to River Elegy, Hong Kong, Chinese University Press, 1997, p. 222. 
46. Ambrose King (Jin Yaoji), Heshang yu Zhongguoren de fansi 河歾与中国人的反思[l'Élégie du Fleuve et la réflexion des Chinois sur eux-mêmes], in Longnian de beican 龙年的悲参 [La Tragédie de l'année du Dragon, Hongkong, Sanlian shudian, p. 62-75.

47. À titre d'exemple : Yi Jianyan, Heshang xuanchuan le shenme? 河㱛宣传了什么? [Que propage l'Élégie du Fleuve ?], Renmin ribao, 19.07.1989. Cet article virulent écrit sous un pseudonyme que l'on peut lire comme « la voix du peuple » ne trouva pas d'éditeur au lendemain de la diffusion de la série. En revanche, dès après le 4 juin, il n'a plus de difficulté à trouver sa place dans les médias officiels.

48. Deux ouvrages recueillent les articles de la polémique en Chine et à l'étranger: Heshang taolunji 河歾讨论集 [Discussions sur L'élégie du Fleuve], Taipei, Fengyun Shidai, 1988 et 1989, et par Cui Wenhua (dir.), Haiwai Heshang dataolun 海外河攻大讨论 [Le grand débat sur L'élégie du fleuve à l'étranger], Harbin, Heilongjiang jiaoyu, 1988. En anglais, voir Su Xiaokang et Wang Luxiang, Deathsong of the River: A Reader's Guide to the Chinese TV Series Heshang, Ithaca, NY: East Asia Program, Cornell University, 1991.

49. Joël Thoraval, «La Tradition rêvée », L'Infini, n³ 30, été 1990, Paris, Gallimard, p. 152

50. Le berceau de la civilisation chinoise n'est pas uniquement le bassin du Fleuve jaune, la Grande muraille n'a jamais empêché les échanges, le développement d'une civilisation marchande et artisanale autour du Fleuve bleu est une réalité... Joël Thoraval, ibid., p. 157.

51. Jean-Pierre Cabestan, Le système politique de la Chine populaire, Paris, PUF, 1994, p. 179-183.

52. Joël Thoraval, op. cit., p. 148.

53. Zhang Lun, La vie intellectuelle en Chine depuis la mort de Mao, Paris, Fayard, 2003, p. 191.

54. Il s'agit d'une transition vers la démocratie dans les mains d'un pouvoir fort pour que règne la stabilité sociale, nécessaire au développement économique. Bien sûr, c'est sur les délais de la transition que se cristallise le débat. Voir Hua Shiping (ed.), Chinese Political Culture, 1989-2000, New-York, ME Sharpe, Studies on Contemporary China, 2001, p. 133-137.

55. Voir les attaques enflammées contre la série de Wang Xiaodong, porte parole du nationalisme chinois sur le blog consacré à la série [http://he-shang.blogspot.com/2007/07/blogpost_01.html] (visité le 27 aout 2008). Ce site réunit l'ensemble des polémiques qui ont suivi la diffusion. Très complet, on y trouve aussi le script et des liens pour télécharger les six épisodes de la série.

56. C'est Wang Jing qui a finement analysé ce « complexe d'infériorité-supériorité » dans son livre High Culture Fever: Politics, Aesthetics, and Ideology in Deng's China, Berkeley,University of California Press, 1996, p. 121-125.

57. Un ouvrage sur le voyage oriental des théoriciens français reste à faire pour répondre au French Theory de François Cusset (op. cit.) qui conte les aventures des travaux de Foucault, Derrida et les autres aux États-Unis et leur incroyable influence sur l'université et la culture américaine. Yue Daiyun y est mentionné page 315. Les premières pages de l'article de Mabel Lee («Walking Out of Other People's Prisons: Liu Zaifu and Gao Xinjian on Chinese Literature in the 1990s ", Asian and African Studies, $\mathrm{n}^{\circ}$ 5, 1996), donnent une idée de l'importance du rôle de Yue Daiyun dans l'introduction de ces théories littéraires au début des années quatre-vingt (p. 98-101). En langue française, Zhang Yinde, professeur à Paris III, semble être un des rares à s'intéresser à la pénétration en Chine de ces théories qui venues de la littérature vont influer tous les champs du savoir pour se retrouver en politique. Voir par exemple « Orient-extrême : Les réinterprétations en Chine des théories postcoloniales ", Revue de littérature comparée, Paris, éditions Klincksieck, $\mathrm{n}$ - 297, 2001/1, p. 133-149.

58. Leur traduction, dès 1987 par Tang Xiaobing 唐小兵(Fredric Jameson, Houxiandai zhuyi yu wenhua lilun 后现代主义与文化理论[Postmodernisme et théories culturelles], Xi'an : Shanxi shifan daxue chubanshe, 1987 ; rééd. Pékin : Beijing daxue chubanshe, 1997, marque le début de la vague postmoderne en Chine, vague qui connaîtra son apogée au milieu des années quatre-vingt-dix en 
la mettant aux mains d'habiles théoriciens comme Zhang Yiwu, Zhang Xudong ou Xu Ben. Pour un aperçu de l'ensemble de la discussion, voir Arif Dirlik et Zhang Xudong, Postmodernism and China, Durham,Duke University Press, 2000. Plus spécifiquement sur les rapports entre histoire et postmodernité, voir Arif Dirlik, "Postmodernism and Chinese History», Boundary 2, 28:3, Durham,Duke University Press, 2001, p. 19-60.

59. Certains intellectuels s'élèvent contre cet usage. Ils ont ainsi inventé le terme de Houxue 后 学, études « post » ou encore postologie, pour réunir sous un même terme ces différents discours. La première occurrence du néologisme apparaît dans les pages de la revue de l'Université chinoise de Hong Kong, $21^{\text {ème }}$ siècle, en février 1995. Dans un article intitulé " "Postologie" et nouveaux conservatismes en Chine»(p.4-15), le sémioticien et publiciste Zhao Yiheng 赵毅衡 vilipende l'agenda conservateur que certains assignent aux théories occidentales. Dans le même numéro, Xu Ben 徐奔dans un article intitulé «La situation de la critique tiers-mondiste en Chine » (p. 16-30) donne un cadre pour que la critique « post » reste positive et stimulante, et ne sombre pas dans un relativisme peu fécond.

60. Zhang Yinde, "Orient-Extrême : les réinterprétations des théories postcoloniales en Chine ", Revue de littérature comparée, $\mathrm{n}^{\circ}$ 297, janvier-mars, 2000, p. 134-135.

61. Ibid., p. 136. Sur le nationalisme contemporain, voir Peter Gries, China's New Nationalism : Pride, Politics and Diplomacy, Berkeley, University of California Press, 2004.

62. Xu Ben, Disenchanted Democracy, Ann Arbor,University of Michigan Press, 1999, p. 18-19.

63. Il existe cependant aujourd'hui un mouvement pour le renouveau du wenyan, langue écrite classique, remplacée au début du XX $\mathrm{XX}^{\mathrm{e}}$ siècle sous la pression de la modernité occidentale, Zhang Yinde, op. cit., p. 140.

64. Leo Ou-fan Lee, Shanghai Modern: The Flowering of a New Urban Culture in China, 1930-1945, Cambridge Mass, Harvard University Press, 1999. Une version abrégée de cet ouvrage sera bientôt publiée en Français aux éditions Robert Laffont.

65. C'est sans doute Wang Hui qui pose le mieux les enjeux de la modernité en Chine au XX siècle dans un texte célèbre qui est devenu l'acte de naissance de cette nébuleuse politique nommée " Nouvelle gauche ». Pour ce faire une idée des enjeux, voir, de Gloria Davies, "Wang Hui, the Historian as Social Critic in China » sur le site : [http://www.overlandexpress.org/182\% 20davies.html]. Le texte de Wang Hui «La pensée contemporaine chinoise et la question de la modernité » a été traduit par Rebecca Karl - en anglais - et édité par Theodore Huters aux Presses universitaires de Harvard en 2003 dans le petit recueil China's New Order. On attend toujours une traduction française.

66. Entretien de Michel Bonnin et Jean-Luc Domenach sur France Inter, le $1^{\mathrm{er}}$ octobre 2009.

67. Tu Wei-Ming, " Historical Consciousness and Cultural Identity ", in History and Memory: Present Reflections on the Past to Build Our Future, The Macau Ricci Institute (ed.), Macau, The Macau Ricci Institute Studies, ${ }^{\circ}$ 5, 2008, p. 16-21.

\section{AUTEUR}

DAVID BARTEL

EHESS-CECMC 